\title{
Chronological set of E. coli 0157:H7 bovine strains establishes a role for repeat sequences and mobile genetic elements in genome diversification
}

\author{
Eliot Stanton ${ }^{1}$, Taylor A. Wahlig ${ }^{1,2}$, Dongjin Park ${ }^{3}$ and Charles W. Kaspar ${ }^{1,4^{*}}$
}

\begin{abstract}
Background: Enterohemorrhagic Escherichia coli 0157:H7 (EHEC) is a significant foodborne pathogen that resides asymptomatically within cattle and other ruminants. The EHEC genome harbors an extensive collection of mobile genetic elements (MGE), including multiple prophage, prophage-like elements, plasmids, and insertion sequence (IS) elements.

Results: A chronological collection of EHEC strains (FRIK804, FRIK1275, and FRIK1625) isolated from a Wisconsin dairy farm (farm X) comprised a closely related clade genetically differentiated by structural alterations to the chromosome. Comparison of the FRIK804 genome with a reference EHEC strain Sakai found a unique prophage like element (PLE, indel 1) and an inversion (1.15 Mb) situated symmetrically with respect to the terminus region. Detailed analysis determined the inversion was due to homologous recombination between repeat sequences in prophage. The three farm X strains were distinguished by the presence or absence of indel $3(61 \mathrm{kbp})$ and indel 4 (48 kbp); FRIK804 contained both of these regions, FRIK1275 lacked indel 4, and indels 3 and 4 were both absent in FRIK1625. Indel 3 was the stx2 prophage and indel 4 involved a deletion between two adjacent prophage with shared repeat sequences. Both FRIK804 and FRIK1275 produced functional phage while FRIK1625 did not, which is consistent with indel 3. Due to their involvement in recombination events, direct and inverted repeat sequences were identified, and their locations mapped to the chromosome. FRIK804 had a greater number and overall length of repeat sequences than E. coli K12 strain MG1655. Repeat sequences were most commonly associated with MGE.

\footnotetext{
* Correspondence: cwkaspar@wisc.edu

'Department of Bacteriology, University of Wisconsin-Madison, Microbial Sciences Building, 1550 Linden Drive, Madison, WI 53706, USA

${ }^{4}$ Food Research Institute, University of Wisconsin-Madison, Microbial Sciences Building, 1550 Linden Drive, Madison, WI 53706, USA

Full list of author information is available at the end of the article
}

(c) The Author(s). 2020 Open Access This article is licensed under a Creative Commons Attribution 4.0 International License, which permits use, sharing, adaptation, distribution and reproduction in any medium or format, as long as you give appropriate credit to the original author(s) and the source, provide a link to the Creative Commons licence, and indicate if changes were made. The images or other third party material in this article are included in the article's Creative Commons licence, unless indicated otherwise in a credit line to the material. If material is not included in the article's Creative Commons licence and your intended use is not permitted by statutory regulation or exceeds the permitted use, you will need to obtain permission directly from the copyright holder. To view a copy of this licence, visit http://creativecommons.org/licenses/by/4.0/. The Creative Commons Public Domain Dedication waiver (http://creativecommons.org/publicdomain/zero/1.0/) applies to the data made available in this article, unless otherwise stated in a credit line to the data. 


\begin{abstract}
(Continued from previous page)
Conclusions: This research demonstrated that three EHEC strains from a Wisconsin dairy farm were closely related and distinguished by variability within prophage regions and other MGE. Chromosome alterations were associated with recombination events between repeat sequences. An inventory of direct and inverted repeat sequences found a greater abundance and total length of repeat sequences in the EHEC strains compared to E. coli strain MG1655. The locations of the repeat sequences were biased towards MGE. The findings from this study expand our understanding of the precise molecular events and elements that contributed to genetic diversification of wild-type EHEC in the bovine and farm environments.
\end{abstract}

Keywords: E. coli O157, st×2, Recombination, Prophage, Direct and inverted repeats

\section{Background}

Enterohemorrhagic E. coli O157:H7 (EHEC) is a significant zoonotic pathogen that causes hemorrhagic colitis and abdominal cramping. In some cases, patients develop hemolytic uremic syndrome (HUS) and kidney failure, particularly in young children [1-3]. Cattle are the primary reservoir of EHEC where residence is asymptomatic [4, 5]. Contaminated ground beef has been associated with transmission from cattle to humans, but an increasing array of foods including leafy greens [6-8], sprouts [9-11], in-shell hazelnuts [12], and cookie dough [13] have been implicated as vehicles in recent outbreaks.

Genomic comparisons of EHEC with nonpathogenic $E$. coli strain MG1655 found a common core sequence interrupted by hundreds of genomic islands [14, 15]. Many of these islands are recognized mobile genetic elements (MGE) including prophage, prophage-like elements (PLE), and insertion sequence (IS) elements. EHEC usually harbor pO157, a $92 \mathrm{kbp}$ F-like plasmid with some genes encoding for virulence factors (i.e., hemolysin) [16, 17]. Other smaller plasmids have been found in some strains [18-20]. EHEC strain Sakai possesses a typical complement of mobile MGE: 18 prophage, 6 PLE, and 80 identified IS, including 19 IS629 elements [15]. By length, prophage account for $11 \%$ of the Sakai chromosome and a majority of MGE. Most of the identified prophage elements are considered incapable of excision or replication and are regarded as cryptic [21]. The genes encoding for Shiga-like toxins Stx1 and Stx2 are located within separate prophage. Stx2 possesses greater cytotoxicity in comparison to Stx1, and Stx 2 production is correlated with the incidence of HUS [22-24]. The stx2-prophage is typically the only functional phage present [21]. Virulence factors located in other MGE also contribute to EHEC pathogenesis $[25,26]$.

EHEC have been divided into distinct lineages based upon octamer-based genome scanning, amplification of lineage-specific polymorphisms, and microarray-based comparative genome hybridization techniques [27-31]. Lineages I (LI) and I/II (LI/II) are isolated from clinical and bovine/environmental sources while lineage II (LII) strains are confined to bovine/environmental sources. This suggests that LII has lower human virulence potential with respect to LI and LI/II. In a previous study, the prophage content of EHEC strains isolated from a Wisconsin dairy farm (farm $\mathrm{X}$ ) was characterized using phage-based PCR markers [32]. Prophage polymorphism profiles (PPP) of strains showed an initial resident LII population supplanted by LI (FRIK804, FRIK1275, and FRIK1625) with strain-specific PPP. Originally distinguished on the basis of differing PFGE profiles, the differences between these strains included the insertional inactivation of $s t x 2$ by IS629 in FRIK1275 and the absence of the stx2-prophage in FRIK1625. FRIK804 contained the $s t x 2$ prophage without IS629. Based on the genomic differences and the date of isolation, FRIK804 likely was the original LI strain on farm $\mathrm{X}$ followed by genomic alterations that resulted in strains FRIK1275 and FRIK1625.

In the current study, whole-genome restriction site mapping and DNA sequencing were used to confirm that the LI strains isolated from farm X were closely related and to discern the molecular events leading to the formation of FRIK1275 and FRIK1625. Prophage and PLE, containing repeat sequences, occupied the sites of chromosomal alterations that distinguished the farm $\mathrm{X}$ strains in most cases. A greater number and overall length of repeat sequences were present in FRIK804 than E. coli strain MG1655. The distribution of repeats was skewed towards MGE. Results from this study highlight the prevalence of repeat sequences, particularly within prophage and PLE, and their role in EHEC diversification in the bovine-farm ecosystem.

\section{Results}

\section{de novo sequence assembly of the FRIK804 genome}

Sequence assembly using Illumina short-read data was hampered by an inability to resolve DNA sequence repeats longer than read length. Draft genomes produced using only short-read data produced fragmented assemblies. Crucially, these assemblies failed to completely capture the assortment of MGE present in the EHEC genome. A high-quality de novo assembly of the 
FRIK804 was produced using single molecule real-time (SMRT) sequence data in conjunction with Illumina paired-end data and confirmation using whole-genome mapping (i.e., optical mapping). The gapless assembly of the FRIK804 genome was required to provide a reference for the other strains analyzed in this study.

Initial assembly of the FRIK804 genome used SPAdes and both SMRT and Illumina data [33]; however, the substitution of two prophage regions was identified and a new assembly was produced using Canu and SMRT data only that lacked this assembly error [34]. Assembly improvement and correction was performed using Pilon [35]. Contigs representing the chromosome and pO157 were identified in the Canu assembly (Table 1). Three small plasmids (pFRIK804-1, pFRIK804-2, and pFRIK804-3) present in the former assembly were absent in the latter suggesting that multiple assembly approaches are useful. pFRIK804-1 was $6.73 \mathrm{kbp}$ and carried genes encoding for colicin D and associated immunity and lysis genes [19]. pFRIK804-2 was $4.09 \mathrm{kbp}$ in length and possessed no predicted phenotype. pFRIK804-3 was $3.31 \mathrm{kbp}$ in length and featured 100\% sequence similarity with pOSAK1, a plasmid previously reported in the genome of EHEC strain Sakai.

\section{Comparative analysis of FRIK804 and Sakai chromosomes} The EHEC strain Sakai was used as a reference for comparison with FRIK804 [15]. The extensive synteny of the two chromosomes was interrupted by a few structural differences. Non-conserved regions consisted of $\mathrm{Mu}$-like prophage with distinct strain-specific integration sites, an inverted segment of the chromosome that included the terminus, and two indels (Fig. 1). Both strains harbored 18 prophage (Ф804-1 - Ф804-18 for FRIK804) (Sp1 - Sp18 for Sakai) while FRIK804 contained 7 PLE (PLE804-1 - PLE804-7) and Sakai 6 PLE (SpLE1 SpLE6)(Table 2 and Fig. 1). Both strains harbored the pO157 plasmid and a $3.31 \mathrm{kbp}$ plasmid pFRIK804-3 (FRIK804) and pOSAK1 (Sakai). IS629 and ISEc8 were the predominate IS in both genomes. Twenty-one IS629 elements were present in FRIK804 and 17 in Sakai (Table S6). Fifteen integration sites for IS629 were shared by the two strains. Nine ISEc 8 elements were present in both strains with 8 common sites of integration (Table S7).

Table 1 FRIK804 genome assembly statistics

\begin{tabular}{llll}
\hline Contig name & Size $(\mathrm{kbp})$ & GC\% & ORFs \\
\hline Chromosome & 5554.24 & 50.52 & 5836 \\
pO157 & 92.70 & 47.59 & 99 \\
pFRIK804-1 & 6.73 & 50.19 & 6 \\
pFRIK804-2 & 4.09 & 49.57 & 3 \\
pFRIK804-3 & 3.31 & 43.42 & 4 \\
\hline
\end{tabular}

Temperate prophage $\mathrm{Mu}$ exhibits transposable activity within the host chromosome [36]. The Mu-like prophage in Sakai (Sp18) is $38.76 \mathrm{kbp}$ in length and is integrated within a putative sorbose operon, disrupting the sorbose operon and specifically locus sorM [37]. Mu-like prophage $\Phi 804-3$ was $39.18 \mathrm{kbp}$ in length and was integrated in an intergenic region separating loci $\operatorname{prpD}$ and prpE. The $\mathrm{Mu}$-like prophage shared $37.97 \mathrm{kbp}$ of (96.52\%) sequence identity (Fig. 2).

Indel-1 (PLE804-1) was a $57.02 \mathrm{kbp}$ region present in FRIK804 and absent in Sakai. Indel-1 disrupted serW encoding for serine tRNA. Alignment of the nucleotide sequence of indel-1 from FRIK804 with the nucleotide sequences of PLE in the Sakai genome (SpLE1-SPLE6) identified common flanking regions shared with SpLE1 (Fig. S1). On this basis, indel-1 was classified as a PLE and designated as PLE804-1. Indel-2 was a $7.46 \mathrm{kbp}$ region present in Sakai but absent in FRIK804 and was not recognized as a MGE. A majority of the $d d p$ operon and $d o s P$ were within this region. The $d d p$ operon contains genes encoding for $\mathrm{D}$-ala-D-ala transport and a dipeptididase $[38,39]$. dos $P$ is a predicted pseudogene.

Comparison of the stx2-prophage in FRIK804 (Ф8046) and Sakai ( $\mathrm{Sp} 5$ ) was conducted due to its central role in human pathogenesis. Sp5 measured $62.71 \mathrm{kbp}$ in length while $\Phi 804-6$ was $61.90 \mathrm{kbp}$ in length. The prophage shared $58.10 \mathrm{kbp}(90.4 \%)$ of common sequence (Fig. 2). Alignment of the prophage was interrupted at several locations; including key phage regulatory regions encoding for repressors CI and Cro, replication proteins $\mathrm{O}$ and $\mathrm{P}$, and anti-terminator $\mathrm{N}$ found in non-conserved regions. Strain Sakai had an IS629 element inserted downstream of stx2 in Sp5 that was absent in $\Phi 804-6$. A broader comparison of $\Phi 804-6$ with other stx2-prophage identified closest sequence homology with phage $933 \mathrm{~W}$, the stx2-prophage present in the genome of EHEC strain EDL933 [40].

An inversion measuring 1.15 Mbp disrupted the alignment of the FRIK804 and Sakai chromosomes. The inverted segment in FRIK804 relative to strain Sakai centered around the terminus of replication region. Sequence motifs associated with termination of replication within the inversion included dif and four Ter sites (TerA, TerB, TerC, and TerD) (Table S1). dif was medially situated with respect to the inversion, resulting in approximate symmetry with respect to both replichores. Replichores 1 and 2 were 2894.4 kbp and $2603.8 \mathrm{kbp}$ in length in Sakai while replichores 1 and 2 in FRIK804 were $2970.9 \mathrm{kbp}$ and $2583.0 \mathrm{kbp}$, respectively. The inversion terminated bilaterally within prophage in both strains. Termini were present within prophage regions Ф804-7 and Ф804-15 in FRIK804, and their chimeric counterparts Sp6 and Sp14 in Sakai (Fig. 3). The sequences of $\Phi 804-7$ and $\Phi 804-15$ were searched for the 


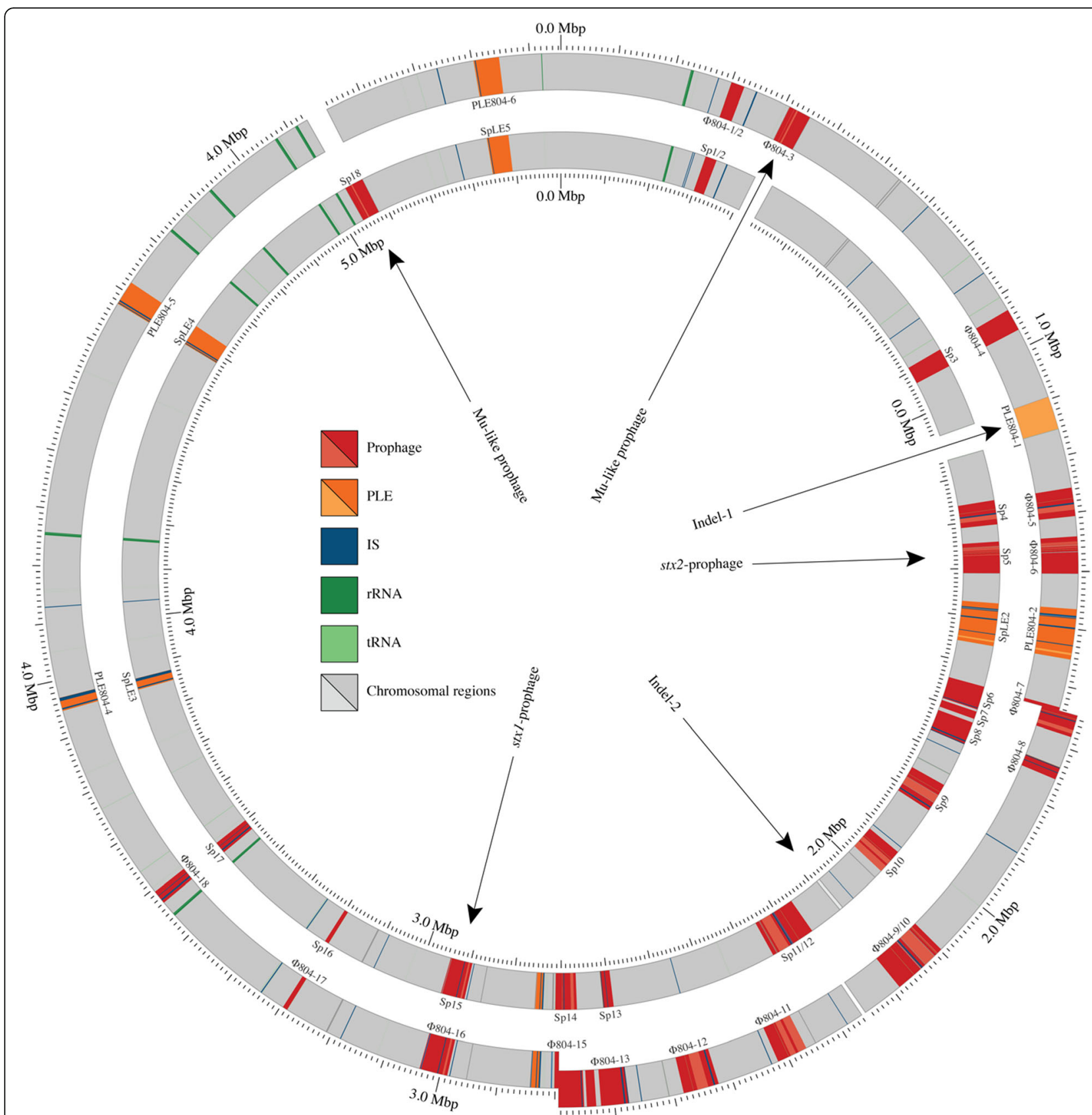

Fig. 1 Comparison of FRIK804 and Sakai chromosomes. Alignment of the FRIK804 (outer) and Sakai (inner) chromosome found disruption of synteny by large-scale structural alterations. To evaluate each dissimilarity, the locations of relevant genomic features (prophage, PLE, IS, rRNA and tRNA) were identified. Non-conserved regions (e.g. indel-1 and indel-2) of each chromosome are shaded lighter relative to conserved regions. Evidence of mosaicism in otherwise conserved prophage was evident in several pairs of homologs, including the stx2-prophage. The majority of dissimilarities that distinguished each chromosome were associated with MGE. A 1.15 Mb inversion is denoted by the offset region in FRIK804. Indel-1 was classified as a PLE and indel-2 was not associated with any MGE. Mu-like prophage were integrated at different loci. The locations of rRNA and tRNA regions are denoted by dark green and light green regions, respectively

presence of repeat sequences greater than $100 \mathrm{bp}$ in length. Sixteen inverted repeat sequences were shared between the prophage (Table S2). A 174 bp repeat sequence precisely flanking the boundaries of the inversion in both $\Phi 804-7 / \Phi 804-15$ and Sp6/Sp14 was identified. To confirm the precise boundaries of the inversion, two pairs of oligonucleotide primers were designed to amplify the repeat sequence and flanking regions in Sp6 (ECs_1507-F/ ECs_1508-R) and Sp14 (ECs_2759-F/ECs_ 2760-R) using PCR (Fig. S1). No amplification was observed using gDNA extracted from FRIK804. Exchange of primers specific to sequences within the inversion 
Table 2 Designation and chromosomal locations of corresponding prophage and PLE in EHEC strains FRIK804 and Sakai. Prophage and PLE were numerically designated in clockwise order (see Fig. 1). The locations of prophage in Sakai were used to identify and locate most prophage and PLE in FRIK804

\begin{tabular}{|c|c|c|c|c|c|c|c|c|}
\hline \multirow[b]{2}{*}{ Name } & \multicolumn{3}{|l|}{ FRIK804 } & \multicolumn{4}{|l|}{ Sakai } & \multirow[b]{2}{*}{ Notes } \\
\hline & Start & End & Length (bp) & Name & Start & End & Length (bp) & \\
\hline Ф804-1 & 300,040 & 310,625 & 10,586 & Sp1 & 300,041 & 310,626 & 10,586 & \\
\hline Ф804-2 & 310,626 & 323,512 & 12,887 & Sp2 & 310,627 & 323,513 & 12,887 & \\
\hline Ф804-3 & 409,092 & 448,271 & 39,180 & & & & & Mu-like prophage ${ }^{a}$ \\
\hline Ф804-4 & 929,716 & 968,301 & 38,586 & Sp3 & 891,123 & 929,708 & 38,586 & \\
\hline PLE804-1 & $1,095,506$ & $1,152,526$ & 57,021 & & & & & \\
\hline Ф804-5 & $1,256,797$ & $1,306,445$ & 49,649 & Sp4 & $1,161,091$ & $1,210,740$ & 49,650 & \\
\hline Ф804-6 & $1,341,717$ & $1,403,616$ & 61,900 & Sp5 & $1,246,012$ & $1,308,719$ & 62,708 & stx2-prophage ${ }^{b}$ \\
\hline PLE804-2 & $1,465,380$ & $1,552,938$ & 87,559 & SpLE1 & $1,370,456$ & $1,456,704$ & 86,249 & \\
\hline Ф804-7 & $1,637,715$ & $1,679,972$ & 42,258 & Sp6 & $1,541,470$ & $1,589,892$ & 48,423 & Inversion terminus \\
\hline Ф804-8 & $1,733,959$ & $1,755,078$ & 21,120 & Sp7 & $1,594,570$ & $1,610,032$ & 15,463 & \\
\hline Ф804-9 & $2,097,886$ & $2,142,115$ & 44,230 & Sp8 & $1,618,153$ & $1,665,049$ & 46,897 & Indel-4 \\
\hline Ф804-10 & $2,142,116$ & $2,187,895$ & 45,780 & Sp9 & $1,757,506$ & $1,815,680$ & 58,175 & Indel-4 \\
\hline Ф804-11 & $2,366,081$ & $2,417,801$ & 51,721 & Sp10 & $1,921,414$ & $1,972,525$ & 51,112 & \\
\hline Ф804-12 & $2,523,534$ & $2,583,605$ & 60,072 & Sp11 & $2,158,174$ & $2,203,951$ & 45,778 & \\
\hline Ф804-13 & $2,675,768$ & $2,722,663$ & 46,896 & Sp12 & $2,203,952$ & $2,250,093$ & 46,142 & \\
\hline Ф804-14 & $2,730,784$ & $2,746,246$ & 15,463 & Sp13 & $2,592,901$ & $2,614,020$ & 21,120 & \\
\hline Ф804-15 & $2,750,924$ & $2,801,116$ & 50,193 & Sp14 & $2,668,007$ & $2,712,035$ & 44,029 & Inversion terminus \\
\hline PLE804-3 & $2,828,472$ & $2,843,243$ & 14,772 & SpLE2 & $2,738,079$ & $2,751,537$ & 13,459 & \\
\hline Ф804-16 & $2,987,650$ & $3,036,836$ & 49,187 & Sp15 & $2,895,926$ & $2,943,804$ & 47,879 & stx1-prophage ${ }^{c}$ \\
\hline Ф804-17 & $3,287,328$ & $3,295,878$ & 8551 & Sp16 & $3,192,983$ & $3,201,533$ & 8551 & \\
\hline Ф804-18 & $3,570,310$ & $3,594,556$ & 24,247 & Sp17 & $3,475,965$ & $3,500,163$ & 24,199 & \\
\hline PLE804-4 & $3,946,431$ & $3,969,884$ & 23,454 & SpLE3 & $3,852,036$ & $3,875,489$ & 23,454 & \\
\hline \multirow[t]{2}{*}{ PLE804-5 } & $4,675,258$ & $4,718,713$ & 43,456 & SpLE4 & $4,580,864$ & $4,624,313$ & 43,450 & \\
\hline & & & & Sp18 & $5,040,843$ & $5,079,601$ & 38,759 & Mu-like prophage ${ }^{a}$ \\
\hline PLE804-6 & $5,402,757$ & $5,412,991$ & 10,235 & SpLE5 & $5,347,085$ & $5,357,319$ & 10,235 & \\
\hline PLE804-7 & $5,413,043$ & $5,447,190$ & 34,148 & SpLE6 & $5,357,371$ & $5,391,518$ & 34,148 & \\
\hline
\end{tabular}

${ }^{\mathrm{a}}$ The $\mathrm{Mu}$-like prophage is capable of transposition

${ }^{b}$ Functional phage

${ }^{\mathrm{C}}$ Does not produce functional phage

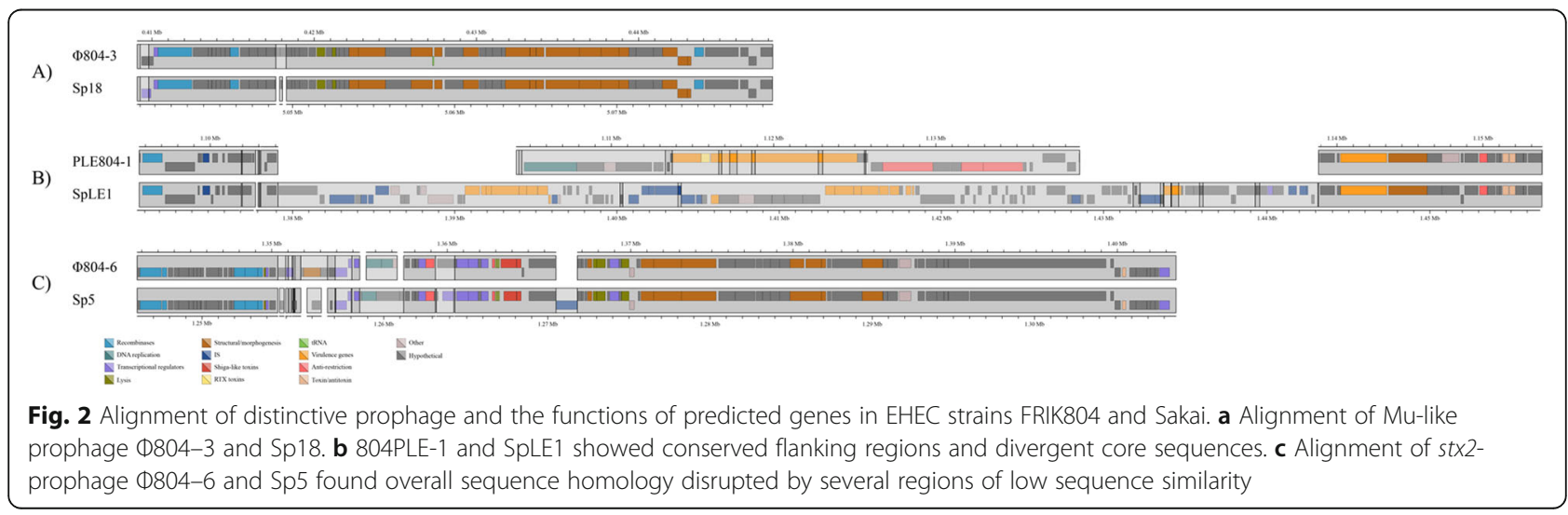




C804-7
Crossover region of homology
Other regions of homology shared between prophage
Predicted ORFs
Fig. 3 Alignment of prophage $\Phi 804-7$ and $\Phi 804-15$ and the locations of 16 inverted and direct repeat sequences, including the flanking ends of
the inversion. Inverted and direct repeat sequences $\geq 100$ bp in length were evaluated as potential sites of recombination (blue). The inverted
segment of the FRIK804 chromosome relative to the Sakai chromosome was flanked by a pair of repeats, the site of the crossover of the
inversion is shown in red. To better show alignment, the orientation of $\Phi 804-15$ is inverted relative to $\Phi 804-7$

(ECs_2760-R/ECs_1508-R and ECs_1507-F /ECs_2759F) resulted in amplification of appropriate size amplicons when using gDNA extracted from FRIK804 only.

\section{Whole-genome mapping}

Whole-genome mapping (also known as optical mapping) produced ordered restriction maps of each farm $\mathrm{X}$ strain. Mapping of the chromosome provided a better understanding of the chromosome rearrangements that distinguished each strain. Whole genome mapping was also valuable for verification of genome assembly of the FRIK804 chromosome. Maps were prepared using the restriction enzyme NcoI. FRIK804, FRIK1275, and FRIK1625 had 559, 548, and 542 fragments, respectively, that were greater than $2.0 \mathrm{kbp}$ in length (Fig. 4a). Based on the sum of the length of the fragments, the

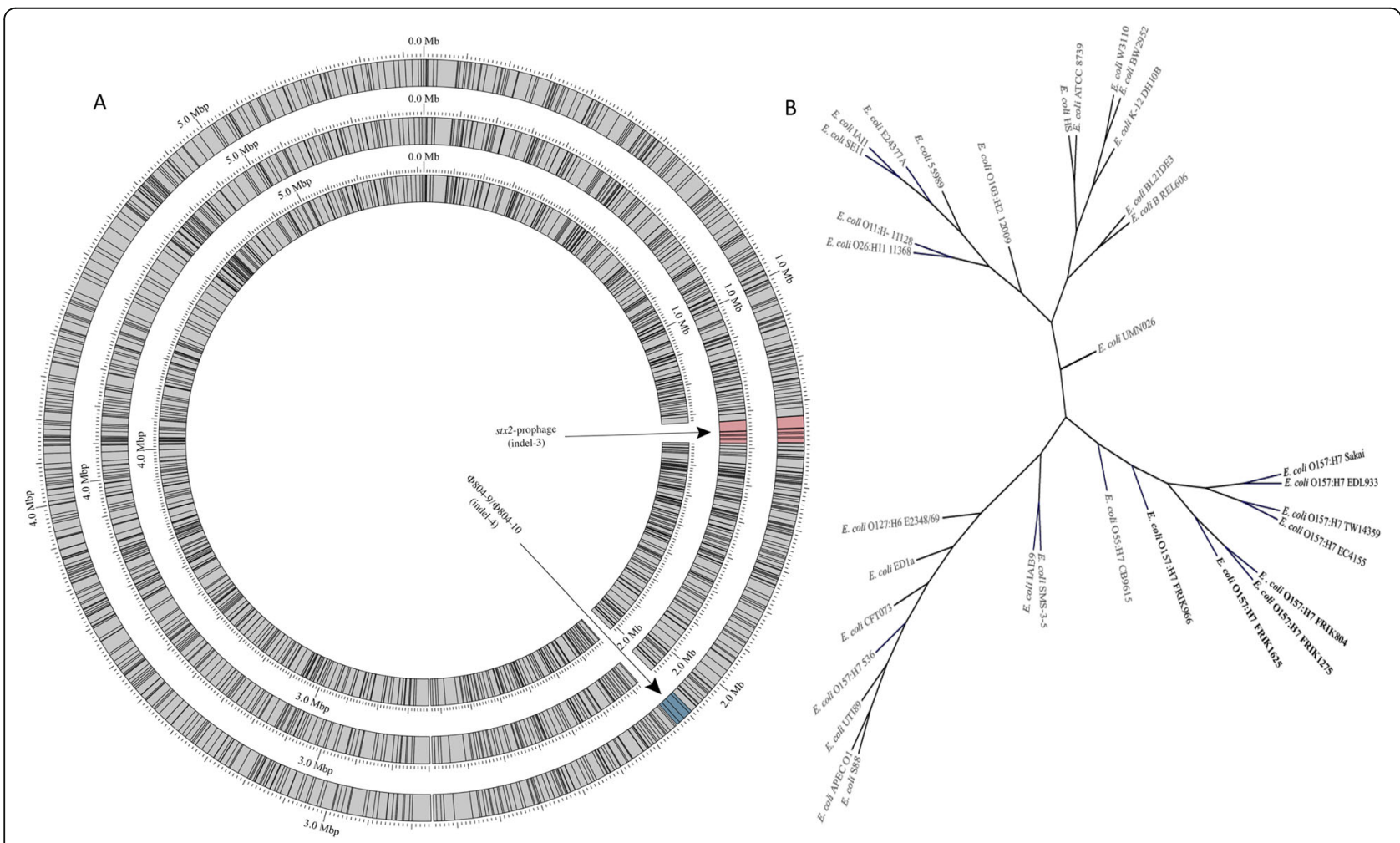

Fig. 4 a Ncol restriction site maps of the chromosomes from FRIK804 (outer), FRIK1275 (middle), and FRIK1625 (inner). An alignment and comparison of farm $X$ strains detected the presence of two indels which distinguished each strain. The identity of each indel was determined using the nucleotide sequence of FRIK804. The location of indel-3 was consistent with the absence of the st×2-prophage in FRIK1625. Indel-4 was determined to overlap portions of two adjacent prophage, $\$ 804-9$ and $\Phi 804-10$. b Hierarchical clustering and pairwise alignment scoring of $\mathrm{Ncol}$ chromosome restriction maps was used to assess relative similarity of the three farm X strains with 30 other E. coli. EHEC O157:H7 strains grouped together (black), and farm X strains (underlined) formed a cluster (bold), indicating that these strains were closely related to one another. FRIK966 is a lineage group II strain included for comparative purposes 
chromosome lengths were estimated to be 5.494 (FRIK804), 5.440 (FRIK1275), and 5.349 (FRIK1625) Mbp. A side-by-side comparison of mapping data from each strain revealed collinear chromosomes disrupted by two indels (indel-3 and indel-4). The presence or absence of these indels served to distinguish each strain. Indel-3 and indel- 4 were estimated to be 61 and $48 \mathrm{kbp}$ in length, respectively. Both indels were present in FRIK804 and absent in FRIK1625. FRIK1275 possessed indel-3 but lacked indel-4. Guided by the nucleotide sequence of FRIK804, the position of indel-3 in FRIK1625 was consistent with the absence of the stx2-prophage. The location of indel-4 corresponded with portions of two adjacent prophage in FRIK804, Ф804-9 and Ф80410. Pairwise alignment scoring of the ordered restriction maps of the three farm X strains and maps of 30 other $E$. coli strains was used to assess similarity via hierarchical clustering. Farm $\mathrm{X}$ strains clustered in a single clade (Fig. 4b).

\section{Plasmid content}

All three farm X strains contained pO157. FRIK804 also contained three smaller plasmids: pFRIK804-1, pFRIK804-2, and pFRIK804-3. Draft genome assemblies were produced using SPAdes with Illumina sequencing data and iteratively polished using Pilon. The FRIK1275 and FRIK1625 assemblies had contigs representing pO157 and pFRIK804-3, but contigs for pFRIK804-1 and pFRIK804-2 were absent.

\section{Inter-prophage deletion in FRIK1275 and FRIK1625}

Whole-genome mapping identified an inter-prophage deletion in adjacent prophage Ф804-9 and Ф804-10 (indel 4) in FRIK1275 and FRIK1625. To precisely determine the boundaries of the absent prophage region in FRIK1275 and FRIK1625, Illumina sequencing data from each strain (including FRIK804) were aligned to the nucleotide sequence of Ф804-9 and Ф804-10 using Bowtie (Fig. 5). Divergence in read coverage was calculated between FRIK804 and both FRIK1275 and FRIK1625. Read coverage found a 47-kbp deletion that spanned prophage Ф804-9 and Ф804-10 in both strains.

Twenty-three direct repeat sequences of $100 \mathrm{bp}$ or greater in length were shared between the two adjacent prophage $\Phi 804-9$ and $\Phi 804-10$ (Table S3). An 822 bp direct repeat was situated at both ends of the region missing in FRIK1275 and FRIK1625. This suggested that homologous recombination between the two repeat sequences was responsible for the deleted region in FRIK1275 and FRIK1625 (indel 4, Fig. 4a). The predicted location and function of the remaining $\Phi 804-9$ and Ф804-10 genes, in FRIK1275 and FRIK1625, aligned with those in FRIK804 (Fig. S3). The 822 bp repeat overlapped with a gene predicted to encode for a phage antirepressor protein (Table S4). PCR amplification of the region was performed using oligonucleotide primers specific to sequences flanking the repeat sequence (ECs_ 2180-int-F and ECs_2272-R). Amplification was observed using gDNA extracted from FRIK1275 and FRIK1625 (Fig. S2). Because of the excessive length, an amplicon was not observed using gDNA extracted from FRIK804 (> 47.7 kbp).

\section{FRIK804 harbors a greater number and overall length of repetitive sequences than nonpathogenic $E$. coli K12 strain MG1655}

The abundance of repeat sequences in the chromosome of FRIK804 was quantified using a custom program written in Perl. Briefly, a sliding-window of 75-mer nucleotide sequences were iteratively hashed to the chromosome coordinate occupied by that sequence. Sequences present in only one location or those lacking a reverse complement in the hash table were discarded. The distribution of repeat sequences was determined using the start and end coordinates of chromosome regions and repeat sequence(s). The categories of chromosome elements were prophage, PLE, IS, rRNA, tRNA, and rearrangement hot spot (Rhs) elements. There were $5,402,917$ unique 75 -mer sequences in the FRIK804 chromosome $(5,554,243 \mathrm{bp}$ in length) in which 112,206 were present two or more times irrespective of

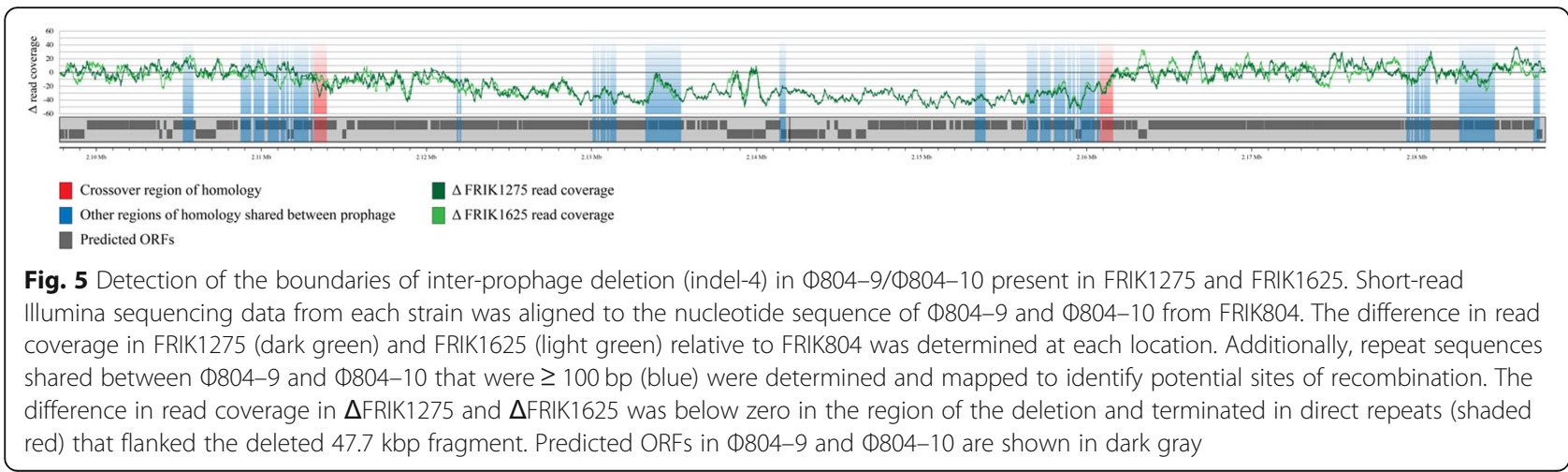


orientation. The majority (67.81\%) of 75-mer repeats were present in prophage and PLE (Fig. 7a), followed by IS (14.58\%) and rRNA (9.01\%). MG1655 possessed fewer repeat sequences overall. The MG1655 chromosome had 38,188 75-mer sequences present more than once and 458,4562 unique sequences (4,641,652 bp in length). There were 24,249 repeat sequences present two or more times, irrespective of orientation. The greatest number of repeats were located within IS (40.45\%) followed by rRNA (30.18\%), prophage (5.42\%), and tRNA (0.15\%).

Repeat sequence complexity was a measure of the repeat copy number irrespective of orientation, i.e. the more times repeat sequences appeared in a chromosome the greater the complexity. Measurement of the copy number of each 75-mer repeat sequence (and disregarding sequence orientation) in each strain found a greater number in FRIK804 compared to MG1655 (Fig. 7b). To further evaluate repeat sequence complexity, the locations of pairs of direct and inverted repeats were defined and termed as links. The number of links for a given direct repeat sequence was a function of $\frac{n_{d}\left(n_{d}-1\right)}{2}$ where $n_{d}$ is the number of direct repeats, and the number of inverted links (reverse complement sequences) was $n_{d} n_{i}$ I, where $n_{i}$ is the number of inverted repeats. The pairs of start and end locations that defined each link were then aligned with their chromosome location. In FRIK804, there were 289,610 direct and 303,420 inverted links. IS accounted for the greatest number of direct links (42.68\%) followed by links within prophage/PLE (34.37\%) and rRNA (15.50\%). IS also accounted for the greatest number of inverted (46.28\%) links followed by prophage/PLE and rRNA (37.65 and 12.96\%, respectively) (Fig. 7b). MG1655 possessed fewer direct (113, $733)$ and inverted $(96,478)$ links. IS accounted for the locations of most direct (51.46\%) and inverted (55.34\%) links followed by rRNA genes (direct $36.23 \%$ and inverted $38.39 \%$ inverted).

The extent and topography of repeat sequences in the chromosome were examined by merging pairs of direct and inverted links that were adjacent to one another, mapping their chromosome locations and connecting links by lines that were plotted using Circos (Fig. 6). Merged links were both more abundant and longer in FRIK804 compared to MG1655 (Fig. 7c). There were 1075 direct and 1241 inverted merged links in FRIK804. The maximum and median direct repeat lengths were 10,011 and $134 \mathrm{bp}$, respectively, and for inverted repeats, the maximum length was 4729 and the median length was $141 \mathrm{bp}$. In MG1655, there were 407 direct and 234 inverted merged links identified. The maximum repeat length for direct repeats was 2816 bp with a median of $144 \mathrm{bp}$, and for inverted repeats, the maximum length was $3024 \mathrm{bp}$ and median was $245 \mathrm{bp}$.

Repetitive regions of the chromosome were defined as areas containing one or more repeat sequences. To evaluate repeat sequences on the basis of length rather

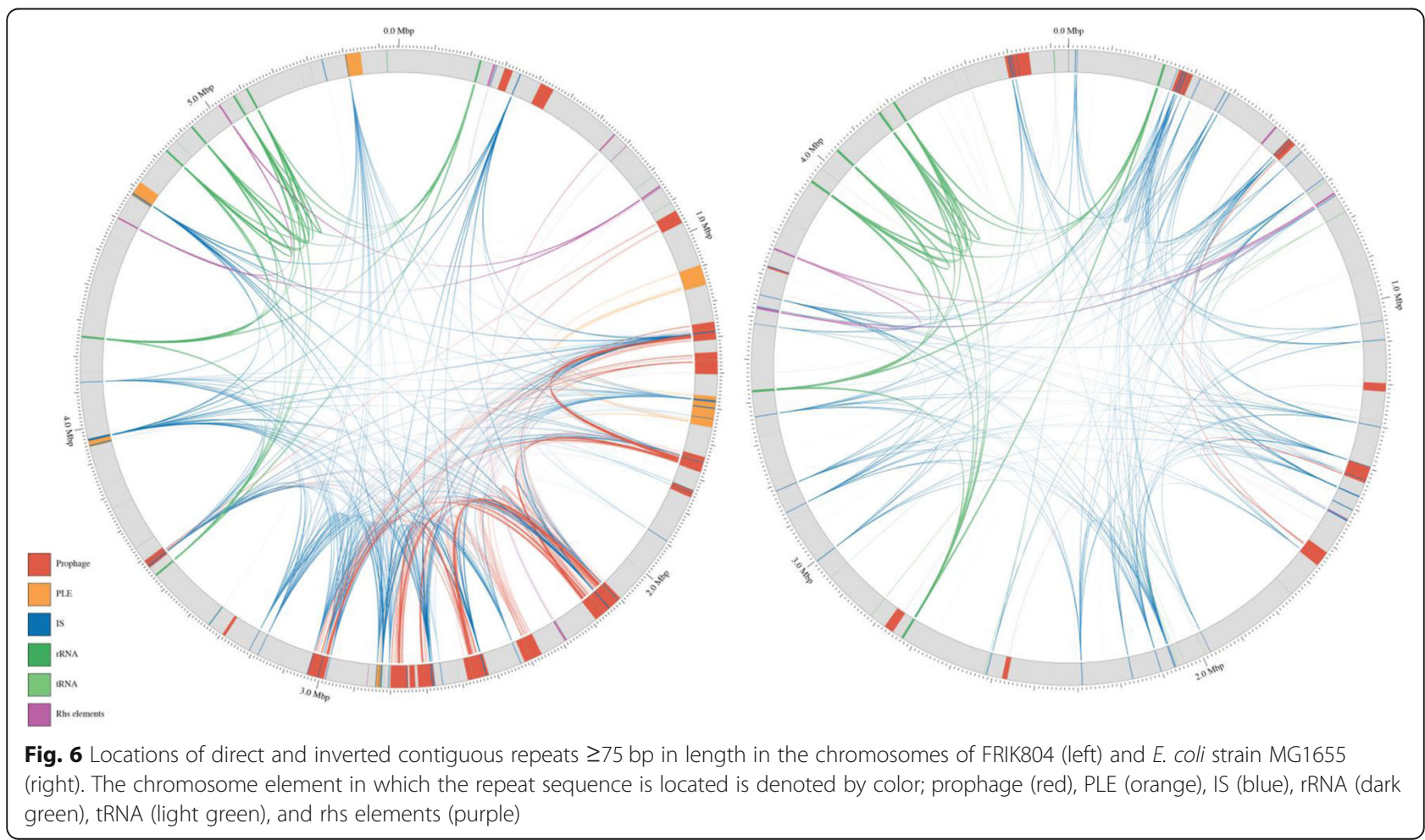




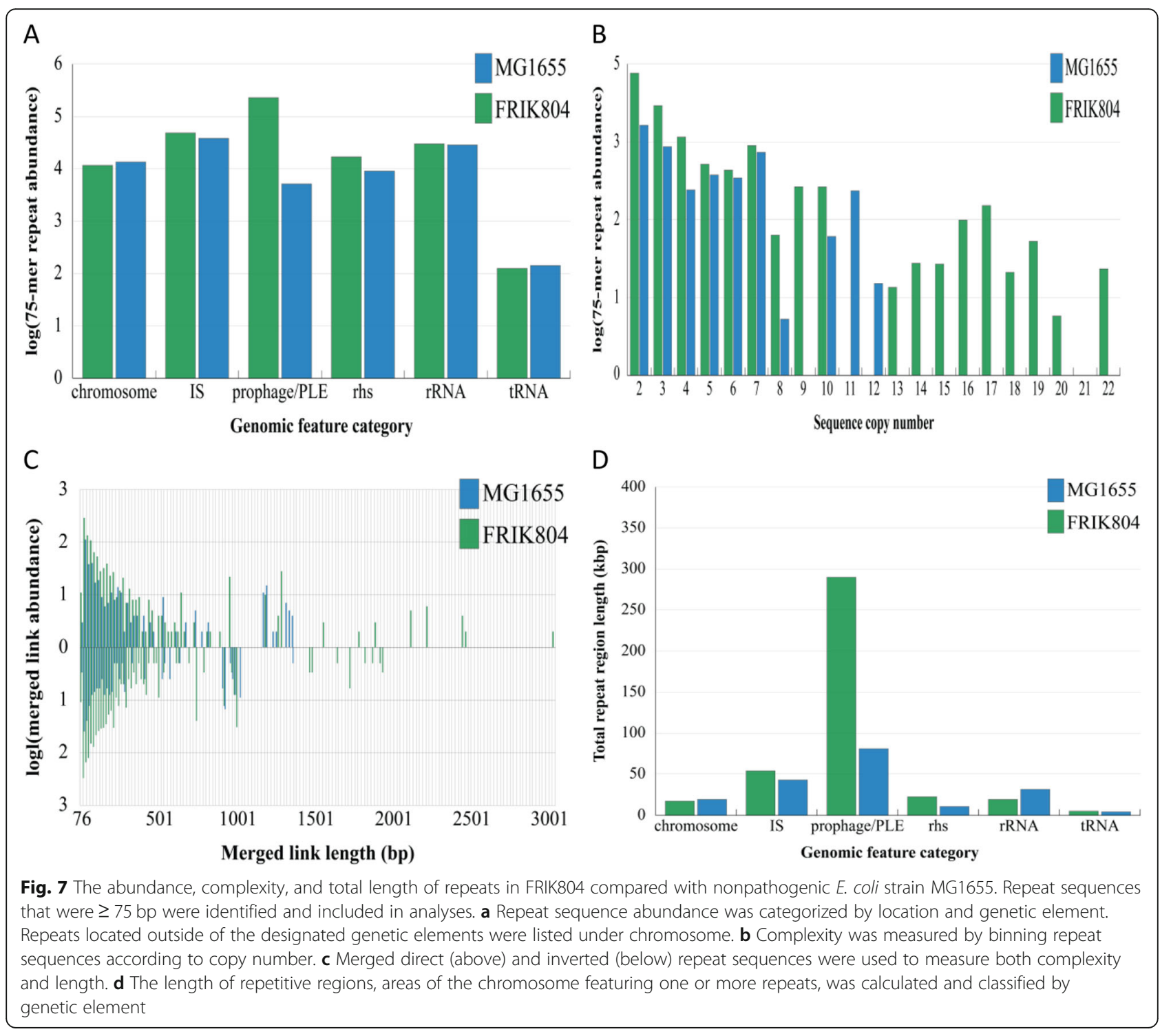

than copy number (e.g., complexity), the length of each annotated chromosome region occupied by repetitive regions were determined. A total of $417,747 \mathrm{bp}(7.52 \%)$ of the FRIK804 chromosome consisted of repetitive regions. These regions were predominantly located within prophage/PLE (5.22\%) (Fig. 7d) followed by IS (0.97\%). Strain MG1655 had a total length of 117,294 bp (2.53\%) of repetitive regions that were most commonly associated with IS $(0.92 \%)$ and rRNA genes $(0.69 \%)$.

\section{stx2-prophage excision site in FRIK1625}

The site of integration of the stx2-prophage is specific in each EHEC lineage [41, 42], with the stx2-prophage integrating into $w r b A$ in LI and I/II strains. Prophage excision requires both Int and excisionase (Xis) activity, resulting in restoration of $a t t P$ and $a t t B$ sites [40]. A putative $a t t B$ site within $w r b A_{\text {EDL933 }}$ was previously identified by Plunkett et al. [40]. Comparison of the nucleotide sequence of $w r b A$ from the FRIK1625 with $w r b A$ from a LI/II strain (without stx2 prophage) found $100 \%$ sequence identity (data not shown). This shows that if the stx2-prophage was present in FRIK1625, excision was mediated by Int/Xis activity rather than homologous recombination, and excision occurred without subsequent lysis of the host.

\section{Detection of stx2 transcript in FRIK1275 (stx2::IS629)}

Identification of different EHEC strains from farm $\mathrm{X}$ was previously determined using XbaI restriction enzyme digest profiles (REDP) generated using PFGE [43]. A majority of EHEC isolates from farm $\mathrm{X}$ during the last year of visits to this farm had a common REDP profile, and FRIK1275 is a representative isolate from this group [43]. PCR amplification of stx2 from strains with this 
common REDP (80 samples) had IS629 inserted in stx2 [32]. Since Stx 2 production and release is linked with prophage induction [44], the farm X strains were tested for transcript of stx 2 and a downstream gene encoding for a putative terminase. Three RT-PCR targets were designed. Primers stx2-US-RT-F/R and stx2-DS-RT-F/R targeted regions of stx 2 immediately upstream and downstream of IS629. The identification of suitable targets downstream of $s t x 2::$ IS629 was hampered by repeat sequences shared between the stx2-prophage and other prophage and PLE in the chromosome; however, a suitable target was identified in a gene annotated as a terminase (primers ECs_1220-RT-F/R). Amplification of a portion of the 16S rRNA gene (primers 16S-RT-F/R) was included as a control. Following prophage induction with MMC, amplification of both stx2-prophage targets and the downstream terminase was detected in RNA extracted from FRIK804 and FRIK1275, demonstrating that IS629 in stx2 did not abolish the production of transcript from stx2 and the downstream terminase in FRIK1275 (Table 3). Amplification using RNA extracted from cultures of FRIK1625 did not result in amplification of targets since it lacked the stx2-prophage.

\section{Discussion}

Epidemiological investigations of EHEC outbreaks have noted REDP variations in strains isolated from implicated foods and clinical stool samples $[45,46]$. The presence of multiple cryptic prophage regions in the EHEC genome are thought to serve as recombination hotspots; however, a detailed understanding of the underlying molecular event(s) that lead to the observed chromosomal alterations is lacking, particularly in isolates from the bovine reservoir $[47,48]$. In this study, a precise examination of chromosome modifications in a chronological set of E. coli $\mathrm{O} 157: \mathrm{H} 7$ strains from a Wisconsin dairy farm (farm X) was conducted. The three strains, each with a unique REDP, belonged to LI and were isolated over a period of approximately 2 years from farm $\mathrm{X}$. FRIK804 was the first E. coli O157:H7 strain isolated from the farm and was found in multiple cattle fecal samples over a two-month period [49]. FRIK1275 was isolated roughly 2 years later than FRIK804 over a 7month period and was recovered from feed, water, and

Table 3 RT-PCR amplification of st×2-prophage markers following induction with mitomycin C

\begin{tabular}{|c|c|c|c|}
\hline \multirow[b]{2}{*}{ Target } & \multicolumn{3}{|l|}{ Strain } \\
\hline & FRIK804 & FRIK1275 & FRIK16215 \\
\hline st×2-US-RT-F/R & + & + & - \\
\hline stx2-DS-RT-F/R & + & + & - \\
\hline ECs_1220-RT-F/R & + & + & - \\
\hline $16 S-F / R$ & + & + & + \\
\hline
\end{tabular}

cattle $[43,49]$. FRIK1625 was isolated from a single fecal sample in the last year of the study. Findings from these analyses found that the presence, absence, and location of MGE, (i.e., plasmids, prophage, and IS elements) accounted for the genomic differences among the strains. Furthermore, direct and inverted repeat sequences commonly found in prophage and PLE in EHEC played a central role in the chromosome changes in the farm X strains.

Analysis of MGE in draft E. coli O157:H7 genomes assembled using short-read DNA sequence data (Illumina) was complicated by repeat sequences found in multiple regions of the chromosome. The assembly of the FRIK804 genome was accomplished using SMRT longread sequencing data and improved using short-read data. Validation of the finished sequence assembly was conducted using whole-genome mapping data (optical mapping). Pairwise alignment of the ordered restriction maps and hierarchical clustering determined the farm $\mathrm{X}$ strains comprised a single clade of strains (Fig. 4b).

Genome diversity in EHEC is associated with MGE $[37,50]$, particularly prophage and PLE. By length, the largest difference between FRIK804 and strain Sakai was a $1.15 \mathrm{Mb}$ inversion in which inverted repeat sequences were identified at the boundaries in a pair of chimeric prophages. The inversion was nearly symmetrical with respect to the axis of replication (defined by dif and oriC). This is important since inversion of the Ter (terminus of replication) region can stall or stop replication forks and induce the SOS response in E. coli [51, 52]. Inversions spanning the terminus of replication region have been found in the chromosomes of EHEC and other enterics and linked to pairs of inverted repeats [48, 53, 54]. The persistence of this clade of strains on farm $\mathrm{X}$, with the inversion relative to strain Sakai, indicates the inversion likely had no or little impact. Other differences were the integration sites of a $\mathrm{Mu}$-like prophage, the presence of an additional PLE in FRIK804, and a $7.46 \mathrm{kbp}$ region not associated with MGE that was present in Sakai and absent in FRIK804 (Fig. 1). Comparison of prophage homologs occupying the same chromosomal site in the two strains identified regions of reduced sequence similarity in otherwise conserved prophage. Exchange of portions of phage genomes by homologous recombination has been previously observed and attributed to phage-encoded recombinases with relaxed fidelity [55-57]. Both FRIK804 and Sakai harbored the pO157 virulence plasmid and a small plasmid (pFRIK804-3) sharing $100 \%$ sequence similarity. FRIK804 possessed two other plasmids (pFRIK804-1 and pFRIK804-2). pFRIK804-1 carried genes for production and immunity to colicin D. No predicted phenotype was ascribed to pFRIK804-2. IS629 was the most numerous recognized IS in both chromosomes. 
Although the locations of a majority of IS629 elements were conserved between the two chromosomes, variability in copy number and location was in agreement with previous reports suggesting relatively high frequencies of transpositional activity $[58,59]$.

Analysis of the three farm $\mathrm{X}$ strains determined that FRIK1275 and FRIK1625 shared a common plasmid profile with Sakai. In addition, FRIK1275 and FRIK1625 shared a common deletion (47.7 Kbp) in two adjacent prophage $\Phi 804-9 / \Phi 804-10$ in comparison to FRIK804 (indel 4, Fig. 4a). The IS629 content of the farm X strains was similar. One important difference noted in FRIK1275 was the insertion of IS629 in stx2 (stx2:: IS629). FRIK1625 lacked the stx2-prophage (indel 3) suggesting non-lethal excision of the $s t x 2$-prophage. Loss of the stx2-prophage has been observed before during laboratory passage $[60,61]$.

Detailed analysis of the 47.7-kbp deletion in FRIK1275 and FRIK1625 was conducted by alignment of shortread sequence data to the intact sequence of adjacent prophage $\Phi 804-9$ and $\Phi 804-10$ from FRIK804. A comparison of the difference in read coverage between strains FRIK1275 and FRIK1625 with that of FRIK804 (no deletion) enabled demarcation of the deletion boundaries (Fig. 5). The difference in read coverage relative to FRIK804 $(<0)$ terminated in direct repeats that flanked the deletion boundaries. Similar deletions in Sakai involving Sp11 and Sp12 in Sakai have been observed in laboratory conditions [62]. The propensity for deletions in this region may be due to the proximity of the two prophages.

Homologous recombination is a process fundamental to DNA replication, repair, and horizontal gene transfer. The frequency of recombination between homologous repeat sequences increases with the length of the repeat in a biphasic manner [63]. The inflection point in this curve is $74 \mathrm{bp}$, below which there is a dramatic decrease in recombination frequency. Based on these findings, Perl scripts were written to detect repeat sequences $\geq 75 \mathrm{bp}$ in length. We did not address approximate repeats in DNA sequences because of the extensive number of homologous sequences present in the O157:H7 genome and the dramatic decrease in the frequency of recombination when mismatches are present within the repeats [63].

The chromosome inversion present in farm X strains relative to the Sakai strain and the partial deletion of Ф804-9/Ф804-10 present FRIK1275 and FRIK1625 both involved repeat sequences. Analysis of direct and inverted repeat sequences $\geq 75 \mathrm{bp}$ was conducted using Perl scripts written to evaluate the abundance, location, and complexity of repeat sequences [GitHub (http:// github.com/eliotstanton/)]. There was a greater abundance of repeat sequences in FRIK804 in comparison to non-pathogenic E. coli K-12 strain MG1655 (Figs. 6 and
7). In FRIK804, the abundance of 75 mer repeat sequences was most prominent in prophage/PLE regions. The complexity of repeat sequences (includes copy number of both direct and inverted repeat sequences) was most commonly associated with IS elements. Analysis of areas of the chromosome containing one or more repeats (repeat regions) found that most repeat regions were located within prophage/PLE. In MG1655, the abundance and complexity of repeat sequences were mostly associated with IS elements. PLE were not identified and comparatively few repeat sequences were located in prophage regions.

IS integration can result in polar mutations [64]. The production of functional phage by FRIK1275 (stx2:: IS629) indicated that genes downstream of $s t \times 2::$ IS629 (encoding for lysis, head, and tail proteins) were expressed. Transcripts from genes upstream and downstream of the stx2::IS629 were detected by RT-PCR although Stx2 was not detected by Western blot [32]. Phage from FRIK1275 (stx2::IS629) formed plaques on host strain MG1655, and PCR amplification of material from individual plaques generated amplicons with a size consistent with the presence of $s t x 2::$ IS629. This indicated that phage production and plaque formation was not the result of excision of IS629 and the restoration of phage function. FRIK1275 (stx2::IS629) was the dominant strain isolated from farm X [43] over a 7-month period of time indicating that Stx2 production was not required for dominance or persistence of EHEC within cattle and the farm environment.

\section{Conclusion}

The results of this study support and illustrate the contribution of MGE (i.e., plasmids, prophage, PLE, and IS) to genome diversity in EHEC from cattle and the farm environment. Detailed analysis of an inversion and inter-prophage deletion provided evidence that homologous recombination between pairs of repeat sequences in prophage were involved in structural alterations to the chromosome. Analysis of repeat sequences in the genome found a greater number and complexity in FRIK804 compared to E. coli K12 strain MG1655 with a preponderance of the repetitive sequences present in MGE. The abundance and location of repeat sequences in FRIK804 may be a driver of chromosome rearrangements in EHEC.

This study contributes to our understanding of the precise molecular events contributing to genomic diversity in wildtype EHEC strains from the bovine and farm environments.

\section{Methods}

\section{Strains}

The EHEC strain Sakai (RIMD 0559952) is a well characterized lineage group I strain that was used as a 
standard reference for comparison purposes (Accession: BA000007.2)(https://doi.org/10.1093/dnares/8.1.11). EHEC strains FRIK804, FRIK1275 and FRIK1625 also belong to lineage group I and were isolated from bovine fecal samples on farm X (PMCID: PMC106160). FRIK966 was used as a representative lineage group II strain isolated from farm $\mathrm{R}$ in Wisconsin [49]. E. coli $\mathrm{K}$ 12 strain MG1655 was from Dr. Tricia Kiley. Stocks of all strains were maintained at $-70^{\circ} \mathrm{C}$ in LB (Luria broth, BD Difco, Houston TX) with $20 \%$ glycerol.

\section{Media and buffers}

LB was used for propagation of E. coli strains. LB agar was used for resuscitation of strains from frozen storage. LB soft agar consisted of LB, agar $(6.0 \mathrm{~g} / \mathrm{L})$ and $\mathrm{CaCl}_{2}$ (10 mM). SM buffer (100 mM NaCl, $8 \mathrm{mM} \mathrm{MgSO}_{4}$, and $50 \mathrm{mM}$ Tris- $\mathrm{HCl}$ ) was used to serially dilute phage lysates. For SMRT sequencing of FRIK804, cells were grown in M9 medium (BD Difco, Houston, TX).

\section{Whole-genome mapping of farm $\mathrm{X}$ strains}

Ordered restriction maps (also known as optical maps) of the chromosomes from farm $\mathrm{X}$ strains were conducted by OpGen (Gaithersburg, MD) using restriction enzyme NcoI as outlined by Zhou et al. [65]. Structural differences in the chromosome of each strain were first resolved by map alignment using Argus MapSolver software. Alignment scoring data of in silico maps of other E. coli and the farm X strains was obtained from MapSolver and used to create a similarity matrix. Hierarchical clustering was performed using UPGMA in $\mathrm{R}$ to create an unrooted tree illustrating the relative similarity of maps from each strain [66].

\section{Illumina sequencing of farm $\mathrm{X}$ strains}

Strains were individually inoculated into LB directly from frozen stock cultures maintained at $-70^{\circ} \mathrm{C}$. Following incubation overnight at $37^{\circ} \mathrm{C}$, cells were harvested by centrifugation. Genomic DNA was prepared using MasterPure Complete DNA and RNA Purification Kit (Epicentre, Madison, WI). Samples were treated with RNAse A (Thermo Fisher Scientific, Waltham, MA) and incubated for $30 \mathrm{~min}$ at $37^{\circ} \mathrm{C}$ to remove RNA. The manufacturer's protocol was modified with regards to precipitation of DNA to include an overnight incubation in $70 \%$ ethanol at $-20^{\circ} \mathrm{C}$. DNA samples were then submitted to the University of Wisconsin-Madison Biotechnology Center. DNA concentration was verified using the Qubit $^{\circ}$ dsDNA HS Assay Kit (Life Technologies, Grand Island, NY). Samples were prepared according to the TruSeq Nano DNA LT Library Prep Kit (Illumina Inc., San Diego, CA) with minor modifications. Samples were sheared using a Covaris M220 Ultrasonicator (Covaris Inc., Woburn, MA), and were size selected for an average insert size of $550 \mathrm{bp}$ using SPRI bead-based size exclusion. The quality and quantity of the finished libraries were assessed using an Agilent High Sensitivity DNA kit and Qubit $^{\circ}$ dsDNA HS Assay Kit, respectively. Libraries were standardized to $2 \mathrm{nM}$, and paired-end $250 \mathrm{bp}$ sequencing was performed using the Illumina MiSeq Sequencer and a MiSeq 500 bp (v2) sequencing cartridge. Images were analyzed using the standard Illumina Pipeline, version 1.8.2.

\section{SMRT sequencing of FRIK804}

FRIK804 was inoculated into M9 media from a single colony on a LB agar plate and incubated overnight at $37^{\circ} \mathrm{C}$. Cells were harvested by centrifugation and washed 4 times using sterile $10 \%$ glycerol. gDNA from washed cell pellets was purified using the method "bacterial genomic DNA isolation using CTAB" from JGI protocol (version 3) (https://jgi.doe.gov/user-programs/pmo-overview/protocols-sample-preparation-information/jgi-bacterial-dna-isolation-ctab-protocol-2012/). The gDNA sample was submitted to the University of WisconsinMilwaukee Great Lakes Genomic Center. A standard Pacific Biosciences large insert library was prepared by fragmenting DNA to approximately $20 \mathrm{~kb}$ using gTUBEs (Covaris, Woburn, MA). Fragmented DNA was enzymatically repaired and ligated to a PacBio adapter to form the SMRTbell Template. Templates larger than 10 $\mathrm{kb}$ were size selected using BluePippin (Sage Science, Beverly, MA). Templates were annealed to a sequence primer, bound to polymerase (P6), and then bound to PacBio Mag-beads and SMRTcell sequenced using a RSII sequencer and C4 chemistry.

\section{Genome assembly}

Draft genome assemblies of each farm X strain were produced using Illumina short-read data and the genome assembler SPAdes 3.11.1 [33]. Corrected paired-end reads were aligned to the assembly using Bowtie 1.1.2 [67]. SAM files were reformatted using Sequence Alignment/Map (SAM) tools (http://samtools.sourceforge. net), and Pilon 1.22 [35] was used to identify and resolve sequence variants. Improvement of the draft assemblies was iteratively performed until no sequence variants were found by Pilon. Contigs smaller than $1.0 \mathrm{~kb}$ or with kmer coverage less than 20 were excluded from final draft assemblies.

The FRIK804 genome was also assembled using PacBio long-read data and Canu 1.7 [34]. Iterative improvement of the assembly was performed as previously outlined. Circularization of the chromosome was performed manually using BLASTn [68-70] to identify overlapping regions. Validation of the assembly was confirmed by generating an in silico whole-genome map of NcoI restriction sites and comparing it to map generated 
from the FRIK804 chromosome to ensure that the two maps were congruent.

\section{Genome annotation and prophage identification} Contigs from the complete FRIK804 genome and draft genomes of FRIK1275 and FRIK1625 were automatically annotated using RAST [71, 72]. Prophage and PLE regions in FRIK804 were identified using the published start and end locations of prophage and PLE in strain Sakai and BLASTn [65].

\section{Nucleotide accession sequence numbers}

The genome sequences of the E. coli O157:H7 strains have been deposited in GenBank; FRIK804 under the accession numbers CP034384-CP034388, FRIK1275 under RWJR00000000 and FRIK1625 under RWJQ00000000.

\section{Whole genome alignment and comparisons}

Alignment of the FRIK804 and Sakai chromosomes was performed using progressiveMauve [73] and BLASTn [68-70]. To better identify common and divergent regions, alignment data from progressiveMauve was formatted using custom Perl scripts to format data for visualization using Circos 0.69 [74]. Common sequence identity shared between genome regions was calculated using the BLAST global alignment interface (Needleman-Wunch). All custom Perl scripts written for this study are available on GitHub (http://github.com/eliotstanton/).

\section{PCR amplification of inversion termini}

The boundaries of the inversion present in strains of the farm $\mathrm{X}$ clade, with respect to Sakai, were verified using oligonucleotide primers ECs_2759-F, ECs_22760-R, ECs_1507-R, and ECs_1508-R. All primers used in this study were manufactured by Integrated DNA technologies (Coralville, IA) and are listed in Table S5. The individual primer pairs ECs_2759-F/ECs_2760-R, ECs_1507F/ECs_1508-R, ECs_2759/ECs_1507-R, and ECs_2760R/ECs_1508-R were separately mixed with gDNA extracted from Sakai, FRIK804, FRIK1275, and FRIK1625. DNA was amplified using rTaq DNA polymerase (Bulldog, Portsmouth, NH) and PCR conditions used were $94{ }^{\circ} \mathrm{C}$ for $5 \mathrm{~min}$, followed by 35 cycles consisting of $94{ }^{\circ} \mathrm{C}$ for $30 \mathrm{~s}, 51^{\circ} \mathrm{C}$ for $30 \mathrm{~s}$, and $72{ }^{\circ} \mathrm{C}$ for $3 \mathrm{~min}$, and concluded by $72{ }^{\circ} \mathrm{C}$ for $5 \mathrm{~min}$. Amplicons were visualized using agarose $(1.0 \%)$ gel electrophoresis and ethidium bromide staining.

PCR amplification of regions of inter-prophage deletions The boundaries of the inter-prophage region present in FRIK804 but absent in FRIK1275 and FRIK1625 was verified using oligonucleotide primers (Table S5). ECs_ 2183-F and ECs_2261-int-R. gDNA extracted from
FRIK804, FRIK1275, and FRIK1625 was amplified using Phusion DNA polymerase (New England Biolabs, Ipswich, MA). PCR conditions used were $98^{\circ} \mathrm{C}$ for $30 \mathrm{~s}$ followed by 30 cycles consisting of $98{ }^{\circ} \mathrm{C}$ for $15 \mathrm{~s}, 66^{\circ} \mathrm{C}$ for $20 \mathrm{~s}$, and $72{ }^{\circ} \mathrm{C}$ for $60 \mathrm{~s}$. PCR was concluded by $72^{\circ} \mathrm{C}$ for $5 \mathrm{~min}$. Amplicons were visualized using agarose $(0.8 \%)$ gel electrophoresis and ethidium bromide staining.

\section{RNA extraction}

In three separate trials, overnight cultures of FRIK804, FRIK1275, and FRIK1625 were incubated overnight at $37^{\circ} \mathrm{C}$. $\mathrm{OD}_{600}$ of overnight cultures was measured and inoculated into fresh $\mathrm{LB}$ at $\mathrm{OD}_{600}=0.01$. Cultures were inoculated in duplicate, to provide a negative control, at $37^{\circ} \mathrm{C}$ with shaking (100 RPM) for $2.25 \mathrm{~h}$. At this point $\mathrm{OD}_{600}$ of cultures was measured prior to addition of mitomycin C (Dot Scientific, Burton, MI) at a final concentration of $1.0 \mu \mathrm{g} / \mathrm{ml}$. Cultures were incubated for one additional hour prior to measuring $\mathrm{OD}_{600}$ of cultures, collection of cells by centrifugation at $4{ }^{\circ} \mathrm{C}$, and disruption of cells by the addition of TRIzol (Thermo Fisher, Waltham, MA). Samples containing TRIzol were stored at $-70^{\circ} \mathrm{C}$ until RNA extraction.

RNA from each frozen TRIzol sample was extracted according to the manufacturer's instructions. Extracted RNA quality and quantity was inspected by measurement of absorbance at $230 \mathrm{~nm}, 260 \mathrm{~nm}$, and $280 \mathrm{~nm}$. Residual DNA contamination was removed using RQ1 DNase (Promega, Madison, WI) in accordance with manufacture's protocol. Following DNase treatment nucleic acid concentration of samples was adjusted to $10 \mathrm{ng} / \mu \mathrm{l}$.

\section{RT-PCR}

Primers (Table S5) targeting regions immediately upstream (stx2-US-RT-F/R) and downstream (stx2-DS-RTF/R) of the IS629 insertion in the FRIK1275 copy of stx2 were used. Primers targeting an additional gene annotated as a phage terminase that was located downstream of stx 2 were also used (ECs_1220-RT-F/R). Amplification of $16 \mathrm{~S}$ rRNA (16S-RT-F/R) was used to provide positive and negative controls. One-step RT-PCR using AccessQuick RT-PCR System (Promega, Madison, WI) was performed consisting of cDNA synthesis at $45^{\circ} \mathrm{C}$ for $45 \mathrm{~min}$ followed by DNA synthesis consisting of $94^{\circ} \mathrm{C}$ for $2 \mathrm{~min}$, and the following cycle conditions $94{ }^{\circ} \mathrm{C}$ for $30 \mathrm{~s}$ and $57^{\circ} \mathrm{C}$ for $30 \mathrm{~s}$. 16S-RT-F/R marker was amplified for 19 cycles and stx2-US-RT-F/R, stx2-US-RT-F/R, and ECs_1220-RT-F/R markers were amplified for 2325 cycles. A final extension step consisting of $68^{\circ} \mathrm{C}$ for 5 min was included for all reactions performed. Amplicons were visualized using agarose (1.5\%) gel electrophoresis and ethidium bromide staining. 
Analysis of IS629 stability during stx2-phage propagation In three separate trials, FRIK1275 was incubated overnight at $37^{\circ} \mathrm{C}$. One $\mathrm{mL}$ of overnight culture was transferred into $9.0 \mathrm{ml}$ of LB broth in $250 \mathrm{~mL}$ Erlenmeyer flasks and incubated at $37^{\circ} \mathrm{C}$ with shaking (100 RPM). Following incubation for $4 \mathrm{~h}$, supernatant containing spontaneously produced phage was collected following centrifugation. Supernatant was sterilized using $0.22 \mu \mathrm{m}$ PVDF filters (Millipore, Burlingame, MA). Concurrently, MG1655 was prepared as a host cell suspension. Upon reaching mid-log phase $\left(\mathrm{OD}_{600}=0.4-0.6\right)$, MG1655 was centrifuged, washed with SM buffer, and resuspended to an $\mathrm{OD}_{600}=2.5$ using SM buffer before storage at $4{ }^{\circ} \mathrm{C}$. Serial dilution of phage lysate was performed using SM buffer. In triplicate, $100 \mu \mathrm{L}$ of each diluted sample was co-incubated with an equal volume of MG1655 cell suspension at $37^{\circ} \mathrm{C}$ for $20 \mathrm{~min}$. Three $\mathrm{ml}$ of soft agar $\left(48^{\circ} \mathrm{C}\right)$ was mixed with each sample and immediately poured onto pre-warmed LB agar plates. Plates were allowed to cool on the bench for $15 \mathrm{~min}$ before overnight incubation at $37^{\circ} \mathrm{C}$.

Twenty-four plaques were picked at random from each trial and material from the plaque was transferred to $10 \mu \mathrm{L}$ of nuclease-free $\mathrm{H}_{2} \mathrm{O}$. DNA was amplified using rTaq DNA polymerase (Bulldog, Portsmouth, $\mathrm{NH}$ ) and stx2a-F/R primers (Table S5). PCR conditions were $94{ }^{\circ} \mathrm{C}$ for $10 \mathrm{~min}$, followed by 30 cycles consisting of $94{ }^{\circ} \mathrm{C}$ for $30 \mathrm{~s}, 53^{\circ} \mathrm{C}$ for $30 \mathrm{~s}$, and $72^{\circ} \mathrm{C}$ for $1 \mathrm{~min}$, amplification was concluded by $72{ }^{\circ} \mathrm{C}$ for $5 \mathrm{~min}$. Amplicons were visualized using agarose $(1.0 \%)$ gel electrophoresis and ethidium bromide staining. The presence or absence of IS629 was determined by amplicon size.

\section{Supplementary information}

Supplementary information accompanies this paper at https://doi.org/10. 1186/s12864-020-06943-x.

\footnotetext{
Additional file 1: Fig. S1. PCR confirmation of inverted repeats present at the flanking ends of the inversion in farm X strains (FRIK804, FRIK1275, and FRIK1625) and control strain Sakai. a Primer pairs ECS_1507-F/ ECs_1508-R and ECs_2759-F/ECs_2760-R were specific to Sp6 and Sp14 in Sakai. Primer pairs ECS_1507-F/ECs_2759-F and ECs_1508-R/ECs_2760-R were specific to regions of $\Phi 804-7$ and $\Phi 804-15$. b Amplification was observed using primer pairs ECs_1507-F/ECs_1508-R (lane 15) and ECs_2759-F/ECs_2760-R (lane 16) using gDNA extracted from Sakai. Amplification was observed using primer pairs ECs_1507-F/ECs_2759-F (lanes 4, 8, and 13) and ECs_1508-R/ECs_2760-R (lanes 5, 9, and 14) using gDNA extracted from farm X strains. Lanes 1 and 10, 1.0-kb ladder.

Additional file 2: Fig. S2. PCR confirmation of regions flanking interprophage deletion in FRIK1275 and FRIK1625 using PCR amplification. Lane 1: 1.0 kb ladder. gDNA in lane 2 (FRIK804), lane 3 (FRIK1275), lane 4 (FRIK1625), and lane 5 (Sakai). Amplification was observed only in strains with the inter-prophage deletion between the identified direct repeats.

Additional file 3: Fig. S3. Predicted function and location of genes in \$804-9 and $\$ 804-10$. The portions of the two adjacent phage in all farm $X$ strain has a shaded grey background. The region in FRIK804 but absent in FRIK1275 and FRIK1625 has a white background.
}

Additional file 4: Table S1. Chromosomal locations of corresponding replication motifs in EHEC FRIK804 and Sakai. Highlighted motifs (light orange) located within the segment of the FRIK804 chromosome that is inverted relative to strain Sakai.

Additional file 5: Table S2. Location and length of inverted repeats in Ф804-7 and \$804-15. Crossover region highlighted in light orange.

Additional file 6: Table S3. Location and length of direct repeats in Ф804-9 and $\$ 804-10$. Crossover region of highlighted in light orange.

Additional file 7: Table S4. Location, classification, and predicted functions of genes in $\Phi 804-9$ and $\$ 804-10$. Highlighted region is present in FRIK804 but absent in FRIK1275 and FRIK1625.

Additional file 8: Table S5. Oligonucleotide primers used in this study. Additional file 9: Table S6. Locations of IS629 elements in FRIK804 and Sakai chromosomes.

Additional file 10: Table S7. Locations of ISEC8 locations in FRIK804 and Sakai chromosomes.

\section{Abbreviations}

BLAST: Basic local alignment search tool; EHEC: Enterohemorrhagic Escherichia coli; FRIK: Food research institute-Kaspar; gDNA: Genomic DNA; LB: Luria broth; MGE: Mobile genetic element; ORF: Open reading frame; PFGE: Pulsed field gel electrophoresis; PacBio: Pacific biosciences; PCR: Polymerase chain reaction; PLE: Prophage-like element; PPP: Prophage polymorphism profile; REDP: Restriction endonuclease digestion profile; RTPCR: Reverse transcriptase-polymerase chain reaction; SMRT: Single molecule real-time; TER: Terminus of replication

\section{Acknowledgements}

We thank Jared Godfrey for technical assistance with PCR analyses and helpful discussions, Dr. Garret Suen for guidance in genome assembly and the development of program scripts, UW-Madison Biotechnology Center for Illumina sequencing, and UW-Milwaukee Great Lakes Genomics Center for Pacific Biosciences RSII Sequencing. Ordered restriction maps (optical maps) of the chromosomes of each farm X strain were determined by OpGen (Gaithersburg, MD).

\section{Authors' contributions}

ES designed the study, preformed experiments, and prepared the draft of the manuscript; TW contributed to gDNA extraction, analysis of DNA sequence data and genome assembly, and contributed to manuscript preparation; DP helped design the experiments and contributed to manuscript preparation; CK coordinated study, analyzed data, wrote portions and reviewed the manuscript. All authors reviewed and approved the manuscript.

\section{Funding}

This work was supported by HATCH grant 142-AAA3661, the Food Research Institute, and the College of Agricultural and Life Sciences at the University of Wisconsin-Madison. E. Stanton was the recipient of the E. Michael and Winona Foster Distinguished Graduate Fellowship.

\section{Availability of data and materials}

The genome sequences of the E. coli O157:H7 strains have been deposited in GenBank; FRIK804 under the accession numbers CP034384-CP034388, FRIK1275 under RWJR00000000 and FRIK1625 under RWJQ00000000. All custom Perl scripts written for this study are available on GitHub (http://github. com/eliotstanton/).

Ethics approval and consent to participate

Not applicable.

\section{Consent for publication}

Not applicable.

\section{Competing interests}

The authors declare that they have no competing interests 


\section{Author details}

'Department of Bacteriology, University of Wisconsin-Madison, Microbial Sciences Building, 1550 Linden Drive, Madison, WI 53706, USA. 'University of Utah, School of Medicine, 30 N 1900 E, Salt Lake City, UT 84132, USA. ${ }^{3}$ Food Science and Technology Department, University of Nebraska-Lincoln, Lincoln, $\mathrm{NE}$, USA. ${ }^{4}$ Food Research Institute, University of Wisconsin-Madison, Microbial Sciences Building, 1550 Linden Drive, Madison, WI 53706, USA.

Received: 11 March 2020 Accepted: 23 July 2020

Published online: 17 August 2020

\section{References}

1. Rangel JM, Sparling PH, Crowe C, Griffin PM, Swerdlow DL. Epidemiology of Escherichia coli 0157:H7 outbreaks, United States, 1982-2002. Emerg Infect Dis. 2005;11:603-9 https://doi.org/10.3201/eid1104.040739.

2. Wachsmuth IK, Griffin PM, Wells JG. Escherichia coli O157:H7, a cause of hemorrhagic colitis and hemolytic uremic syndrome. Acta Paediatr Jpn. 1991;33:603-12 https://doi.org/10.1111/j.1442-200x.1991.tb01872.x.

3. Riley LW, Remis RS, Helgerson SD, Mcgee HB, Wells JG, Davis BR, et al. Hemorrhagic colitis associated with a rare Escherichia coli serotype. N Engl J Med. 1983;308:681-5.

4. Borczyk AA, Karmali MA, Lior H, Duncan LMC. Bovine reservoir for Verotoxinproducing Escherichia Coli 0157:H7. Lancet. 1987;329:98.

5. Ferens WA, Hovde CJ. Escherichia coli O157:H7: animal reservoir and sources of human infection. Foodborne Pathog Dis. 2011;8:465-87 https:// doi.org/10.1089/fpd.2010.0673.

6. Marder EP, Garman KN, Ingram LA, Dunn JR. Multistate outbreak of escherichia coli 0157:H7 associated with bagged salad. Foodborne Pathog Dis. 2014;11:593-5.

7. Wendel AM, Hoang Johnson D, Sharapov U, Grant J, Archer JR, Monson T, et al. Multistate outbreak of Escherichia coli O157:H7 infection associated with consumption of packaged spinach, august-September 2006: the Wisconsin investigation. Clin Infect Dis. 2009;48:1079-86 https://doi.org/10. 1086/597399.

8. Grant J, Wendelboe AM, Wendel A, Jepson B, Torres P, Smelser C, et al. Spinach-associated Escherichia coli 0157:H7 outbreak, Utah and New Mexico, 2006. Emerg Infect Dis. 2008;14:1633-6.

9. Michino H, Araki K, Minami S, Takaya S, Sakai N, Miyazaki M, et al. Massive outbreak of Escherichia coli 0157: H7 infection in schoolchildren in Sakai City, Japan, associated with consumption of white radish sprouts. Am J Epidemiol. 1999;150:787-96 https://doi.org/10.1093/oxfordjournals.aje.a010082.

10. Ferguson DD, Scheftel J, Cronquist A, Smith K, Woo-Ming A, Anderson E, et al. Temporally distinct Escherichia coli 0157 outbreaks associated with alfalfa sprouts linked to a common seed source - Colorado and Minnesota, 2003. Epidemiol Infect. 2005;133:439-47 https://doi.org/10.1017/ S0950268804003589.

11. Breuer T, Benkel DH, Shapiro RL, Hall WN, Winnett MM, Linn MJ, et al. A Multistate Outbreak of Escherichia coli 0157:H7 Infections Linked to Alfalfa Sprouts Grown from Contaminated Seeds. Emerg Infect Dis J - CDC. 2001;7: $6 \mathrm{https}: / /$ doi.org/10.3201/eid0706.010609.

12. Miller BD, Rigdon CE, Ball J, Rounds JM, Klos RF, Brennan BM, et al. Use of traceback methods to confirm the source of a multistate escherichia coli 0157:H7 outbreak due to in-shell hazelnuts. J Food Prot. 2012;75:320-7.

13. Neil KP, Biggerstaff G, MacDonald JK, Trees E, Medus C, Musser KA, et al. A novel vehicle for transmission of Escherichia coli O157:H7 to humans: multistate outbreak of E. coli 0157:H7 infections associated with consumption of ready-to-bake commercial prepackaged cookie dough--United States, 2009. Clin Infect Dis. 2012;54:511-8 https://doi.org/10.1093/cid/cir831.

14. Perna NT, Plunkett G, Burland V, Mau B, Glasner JD, Rose DJ, et al. Genome sequence of enterohaemorrhagic Escherichia coli 0157:H7. Nature. 2001; 409:529-33 https://doi.org/10.1038/35054089.

15. Hayashi T, Makino K, Ohnishi M, Kurokawa K, Ishii K, Yokoyama K, et al. Complete genome sequence of enterohemorrhagic Eschelichia coli O157: $\mathrm{H} 7$ and genomic comparison with a laboratory strain K-12. DNA Res. 2001;8: 11-22 https://doi.org/10.1093/dnares/8.1.11.

16. Bauer ME, Welch RA. Characterization of an RTX toxin from enterohemorrhagic Escherichia coli 0157:H7. Infect Immun. 1996;64:167-75.

17. Schmidt $H$, Beutin $L$, Karch H. Molecular analysis of the plasmid-encoded hemolysin of Escherichia coli O157:H7 strain EDL 933. Infect Immun. 1995; 63:1055-61.
18. Makino K. Complete Nucleotide Sequences of 93-kb and 3.3-kb Plasmids of an Enterohemorrhagic Escherichia coli 0157:H7 Derived from Sakai Outbreak. DNA Res. 1998:5:1-9 https://doi.org/10.1093/dnares/5.1.1.

19. Hofinger $C$, Karch $H$, Schmidt $H$. Structure and function of plasmid pColD157 of enterohemorrhagic Escherichia coli $\mathrm{O} 157$ and its distribution among strains from patients with diarrhea and hemolytic-uremic syndrome. J Clin Microbiol. 1998;36:24-9.

20. Ratnam S, March SB, Ahmed R, Bezanson GS, Kasatiya S. Characterization of Escherichia coli serotype 0157:H7. J Clin Microbiol. 1988;26:2006-12 http:// www.ncbi.nlm.nih.gov/pubmed/3053758. Accessed 16 Feb 2020.

21. Asadulghani M, Ogura Y, Ooka T, Itoh T, Sawaguchi A, Iguchi A, et al. The defective prophage pool of Escherichia coli 0157: prophage-prophage interactions potentiate horizontal transfer of virulence determinants. PLoS Pathog. 2009;5:e1000408 https://doi.org/10.1371/journal.ppat.1000408.

22. Boerlin P, McEwen SA, Boerlin-Petzold F, Wilson JB, Johnson RP, Gyles CL. Associations between virulence factors of Shiga toxin-producing Escherichia coli and disease in humans. J Clin Microbiol. 1999;37:497-503 https://doi. org/10.1128/jcm.37.3.497-503.1999.

23. Donohue-Rolfe A, Kondova I, Oswald S, Hutto D, Tzipori S, Donohue-Rolfe A et al. Escherichia coli 0157:H7 strains that express Shiga toxin (Stx) 2 alone are more neurotropic for Gnotobiotic piglets than are Isotypes producing only Stx1 or both Stx1 and Stx2. J Infect Dis. 2000;181:1825-9 https://doi. org/10.1086/315421.

24. Fuller CA, Pellino CA, Flagler MJ, Strasser JE, Weiss AA. Shiga toxin subtypes display dramatic differences in potency. Infect Immun. 2011;79:1329-37 https://doi.org/10.1128/IAl.01182-10.

25. Gruenheid S, Sekirov I, Thomas NA, Deng W, O'Donnell P, Goode D, et al. Identification and characterization of NleA, a non-LEE-encoded type II translocated virulence factor of enterohaemorrhagic Escherichia coli 0157: H7. Mol Microbiol. 2004;51:1233-49 https://doi.org/10.1046/j.1365-2958.2003. 03911.x

26. Tobe T, Beatson SA, Taniguchi H, Abe H, Bailey CM, Fivian A, et al. An extensive repertoire of type III secretion effectors in Escherichia coli 0157 and the role of lambdoid phages in their dissemination. Proc Natl Acad Sci U S A. 2006;103:14941-6 https://doi.org/10.1073/pnas.0604891103.

27. Kim J, Nietfeldt J, Benson AK. Octamer-based genome scanning distinguishes a unique subpopulation of Escherichia coli O157:H7 strains in cattle. Proc Natl Acad Sci U S A. 1999;96:13288-93 https://doi.org/10.1073/ pnas.96.23.13288.

28. Kim J, Nietfeldt J, Ju J, Wise J, Fegan N, Desmarchelier P, et al. Ancestral divergence, genome diversification, and phylogeographic variation in subpopulations of sorbitol-negative, $\beta$-glucuronidase-negative enterohemorrhagic Escherichia coli O157. J Bacteriol. 2001;183:6885-97 https://doi.org/10.1128/JB.183.23.6885-6897.2001.

29. Yang Z, Kovar J, Kim J, Nietfeldt J, Smith DR, Moxley RA, et al. Identification of common subpopulations of non-sorbitol-fermenting, beta-glucuronidasenegative Escherichia coli 0157:H7 from bovine production environments and human clinical samples. Appl Environ Microbiol. 2004;70:6846-54 https://doi.org/10.1128/AEM.70.11.6846-6854.2004.

30. Zhang Y, Laing C, Steele M, Ziebell K, Johnson R, Benson AK, et al. Genome evolution in major Escherichia coli 0157:H7 lineages. BMC Genomics. 2007; 8:121 https://doi.org/10.1186/1471-2164-8-121.

31. Laing CR, Buchanan C, Taboada EN, Zhang Y, Karmali MA, Thomas JE, et al. In silico genomic analyses reveal three distinct lineages of Escherichia coli 0157:H7, one of which is associated with hyper-virulence. BMC Genomics. 2009;10:287 https://doi.org/10.1186/1471-2164-10-287.

32. Park D, Stanton E, Ciezki K, Parrell D, Bozile M, Pike D, et al. Evolution of the Stx2-encoding Prophage in persistent bovine Escherichia coli 0157:H7 strains. Appl Environ Microbiol. 2013;79:1563-72 https://doi.org/10.1128/ AEM.03158-12.

33. Bankevich A, Nurk S, Antipov D, Gurevich AA, Dvorkin M, Kulikov AS, et al. SPAdes: a new genome assembly algorithm and its applications to single-cell sequencing. J Comput Biol. 2012;19:455-77 https://doi.org/10.1089/cmb.2012.0021.

34. Koren S, Walenz BP, Berlin K, Miller JR, Bergman NH, Phillippy AM. Canu: scalable and accurate long-read assembly via adaptive $\mathrm{K}$-mer weighting and repeat separation. Genome Res. 2017;27:722-36.

35. Walker BJ, Abeel T, Shea T, Priest M, Abouelliel A, Sakthikumar S, et al. Pilon: an integrated tool for comprehensive microbial variant detection and genome assembly improvement. PLoS One. 2014;9.

36. Harshey RM. Transposable Phage Mu. Microbiol Spectr. 2014;2. https://doi. org/10.1128/microbiolspec.MDNA3-0007-2014. 
37. Ohnishi M, Terajima J, Kurokawa K, Nakayama K, Murata T, Tamura K, et al. Genomic diversity of enterohemorrhagic Escherichia coli 0157 revealed by whole genome PCR scanning. Proc Natl Acad Sci U S A. 2002;99:17043-8 https://doi.org/10.1073/pnas.262441699.

38. Magasanik B. Global regulation of gene expression. Proc Natl Acad Sci U S A. 2000;97:14044-5.

39. Zimmer DP, Soupene E, Lee HL, Wendisch VF, Khodursky AB, Peter BJ, et al. Nitrogen regulatory protein C-controlled genes of Escherichia coli: scavenging as a defense against nitrogen limitation. Proc Natl Acad Sci U S A. 2000;97:14674-9.

40. Plunkett G, Rose DJ, Durfee TJ, Blattner FR. Sequence of Shiga toxin 2 phage 933W from Escherichia coli 0157:H7: Shiga toxin as a phage lategene product. J Bacteriol. 1999;181:1767-78 https://doi.org/10.1128/jb.181.6. 1767-1778.1999.

41. Shaikh N, Tarr PI. Escherichia coli O157:H7 Shiga toxin-encoding bacteriophages: integrations, excisions, truncations, and evolutionary implications. J Bacteriol. 2003;185:3596-605 https://doi.org/10.1128/JB.185. 12.3596-3605.2003.

42. Eppinger M, Mammel MK, Leclerc JE, Ravel J, Cebula TA. Genomic anatomy of Escherichia coli O157:H7 outbreaks. Proc Natl Acad Sci U S A. 2011;108: 20142-7 https://doi.org/10.1073/pnas.1107176108.

43. Shere JA, Bartlett KJ, Kaspar CW. Longitudinal study of Escherichia coli O157: H7 dissemination on four dairy farms in Wisconsin. Appl Envir Microbiol. 1998;64:1390-9 https://doi.org/10.1128/aem.64.4.1390-1399.1998.

44. Wagner PL, Neely MN, Zhang X, Acheson DW, Waldor MK, Friedman DI. Role for a phage promoter in Shiga toxin 2 expression from a pathogenic Escherichia coli strain. J Bacteriol. 2001;183:2081-5 https://doi.org/10.1128/ JB.183.6.2081-2085.2001.

45. Gouveia S, Proctor ME, Lee MS, Luchansky JB, Kaspar CW. Genomic comparisons and Shiga toxin production among Escherichia coli 0157:H7 isolates from a day care center outbreak and sporadic cases in southeastern Wisconsin. J Clin Microbiol. 1998;36:727-33 http://www.ncbi.nlm.nih.gov/ pubmed/9508303. Accessed 18 Feb 2020.

46. Welinder-Olsson C, Stenqvist K, Badenfors M, Brandberg Å, Florén K, Holm $M$, et al. EHEC outbreak among staff at a children's hospital - use of PCR for verocytotoxin detection and PFGE for epidemiological investigation. Epidemiol Infect. 2004;132:43-9 https://doi.org/10.1017/S0950268803001444.

47. Kotewicz ML, Jackson SA, LeClerc JE, Cebula TA. Optical maps distinguish individual strains of Escherichia coli O157 : H7. Microbiology. 2007;153:172033 https://doi.org/10.1099/mic.0.2006/004507-0.

48. Iguchi A, lyoda S, Terajima J, Watanabe H, Osawa R. Spontaneous recombination between homologous prophage regions causes large-scale inversions within the Escherichia coli O157:H7 chromosome. Gene. 2006; 372:199-207 https://doi.org/10.1016/j.gene.2006.01.005

49. Faith NG, Shere JA, Brosch R, Arnold KW, Ansay SE, Lee MS, et al. Prevalence and clonal nature of Escherichia coli 0157:H7 on dairy farms in Wisconsin. Appl Environ Microbiol. 1996;62.

50. Kudva IT, Evans PS, Perna NT, Barrett TJ, Ausubel FM, Blattner FR, et al. Strains of Escherichia coli 0157:H7 differ primarily by insertions or deletions, not single-nucletide polymorphisms. J Bacteriol. 2002;184:1873-9 https://doi. org/10.1128/JB.184.7.1873-1879.2002.

51. Sharma B, Hill TM. Insertion of inverted Ter sites into the terminus region of the Escherichia coli chromosome delays completion of DNA replication and disrupts the cell cycle. Mol Microbiol. 1995;18:45-61 https://doi.org/10.1111/ j.1365-2958.1995.mmi_18010045.x.

52. Bidnenko V. Replication fork collapse at replication terminator sequences. EMBO J. 2002;21:3898-907 https://doi.org/10.1093/emboj/cdf369.

53. Alokam S, Liu S-L, Said K, Sanderson KE. Inversions over the terminus region in Salmonella and Escherichia coli: IS200s as the sites of homologous recombination inverting the chromosome of Salmonella enterica serovar typhi. J Bacteriol. 2002;184:6190-7 https://doi.org/10.1128/JB.184.22.6190-6197.2002.

54. Wang D, Li S, Guo F, Ning K, Wang L. Core-genome scaffold comparison reveals the prevalence that inversion events are associated with pairs of inverted repeats. BMC Genomics. 2017;18 https://doi.org/10.1186/s12864017-3655-0.

55. De Paepe M, Hutinet G, Son O, Amarir-Bouhram J, Schbath S, Petit M-A Temperate phages acquire DNA from defective prophages by relaxed homologous recombination: the role of rad52-like recombinases. PLoS Genet. 2014;10:e1004181 https://doi.org/10.1371/journal.pgen.1004181.

56. Johansen BK, Wasteson Y, Granum PE, Brynestad S. Mosaic structure of Shiga-toxin-2-encoding phages isolated from Escherichia coli O157:H7 indicates frequent gene exchange between lambdoid phage genomes. Microbiology. 2001;147:1929-36 http://mic.sgmjournals.org/content/147/7/1 929.short. Accessed 27 Mar 2014.

57. Juhala RJ, Ford ME, Duda RL, Youlton A, Hatfull GF, Hendrix RW. Genomic sequences of bacteriophages HK97 and HK022: pervasive genetic mosaicism in the lambdoid bacteriophages. J Mol Biol. 2000;299:27-51.

58. Stanton E, Park D, Döpfer D, Ivanek R, Kaspar CWCW. Phylogenetic characterization of Escherichia coli 0157:H7 based on IS629 distribution and Shiga toxin genotype. Microbiology. 2014;160(PART 3):502-5013 https://doi. org/10.1099/mic.0.073437-0.

59. Rump LV, Fischer M, González-Escalona N. Different IS629 transposition frequencies exhibited by Escherichia coli O157:H7 strains in the stepwise evolutionary model. Appl Environ Microbiol. 2011;77:5030-3 https:/doi.org/ 10.1128/AEM.00249-11.

60. Kulow MJ, Gonzales TK, Pertzborn KM, Dahm J, Miller BA, Park D, et al. Differences in colonization and shedding patterns after oral challenge of cattle with three Escherichia coli 0157:H7 strains. Appl Environ Microbiol. 2012;78:8045-55 https://doi.org/10.1128/AEM.02363-12.

61. Karch H, Meyer T, Russmann H, Heesemann J. Frequent loss of Shiga-like toxin genes in clinical isolates of Escherichia coli upon subcultivation downloaded from. 1992. http://iai.asm.org/. Accessed 19 Feb 2020.

62. Chen C, Lewis CR, Goswami K, Roberts EL, DebRoy C, Dudley EG. Identification and characterization of spontaneous deletions within the Sp11-Sp12 prophage region of Escherichia coli 0157:H7 Sakai. Appl Environ Microbiol. 2013;79:1934-41 https://doi.org/10.1128/AEM.03682-12.

63. Watt VM, Ingles CJ, Urdea MS, Rutter WJ. Homology requirements for recombination in Escherichia coli. Proc Natl Acad Sci U S A. 1985;82:4768-22 https://doi.org/10.1073/pnas.82.14.4768.

64. Hutchison CA, Merryman C, Sun L, Assad-Garcia N, Richter RA, Smith HO, et al. Polar effects of transposon insertion into a minimal bacterial genome. J Bacteriol. 2019:201.

65. Zhou Z, Li X, Liu B, Beutin L, Xu J, Ren Y, et al. Derivation of Escherichia coli 0157:H7 from its O55:H7 precursor. PLoS One. 2010;5:e8700. https://doi.org/ 10.1371/journal.pone.0008700.

66. Team RC. R: a language and environment for statistical computing. 2017. https://www.r-project.org/.

67. Langmead B, Trapnell C, Pop M, Salzberg SL, Down T, Rakyan V, et al. Ultrafast and memory-efficient alignment of short DNA sequences to the human genome. Genome Biol. 2009;10:R25 https://doi.org/10.1186/gb-200910-3-r25.

68. Agarwala R, Barrett T, Beck J, Benson DA, Bollin C, Bolton E, et al. Database resources of the National Center for biotechnology information. Nucleic Acids Res. 2017:45:D12-7.

69. Camacho C, Coulouris G, Avagyan V, Ma N, Papadopoulos J, Bealer K, et al. BLAST+: architecture and applications. BMC Bioinformatics. 2009;10:421 https://doi.org/10.1186/1471-2105-10-421.

70. Madden T. The BLAST sequence analysis tool. 2003. https://www.ncbi.nlm. nih.gov/books/NBK21097/. Accessed 13 Dec 2018.

71. Overbeek R, Olson R, Pusch GD, Olsen GJ, Davis JJ, Disz T, et al. The SEED and the rapid annotation of microbial genomes using subsystems technology (RAST). Nucleic Acids Res. 2014;42(Database issue):D206-14. https://doi.org/10.1093/nar/gkt1226.

72. Aziz RK, Bartels D, Best AA, DeJongh M, Disz T, Edwards RA, et al. The RAST server: rapid annotations using subsystems technology. BMC Genomics. 2008;9:75. https://doi.org/10.1186/1471-2164-9-75.

73. Darling ACE, Mau B, Blattner FR, Perna NT. Mauve: multiple alignment of conserved genomic sequence with rearrangements. Genome Res. 2004;14: 1394-403 https://doi.org/10.1101/gr.2289704.

74. Krzywinski M, Schein J, Birol I, Connors J, Gascoyne R, Horsman D, et al. Circos: an information aesthetic for comparative genomics. Genome Res. 2009;19:1639-45 https://doi.org/10.1101/gr.092759.109.

\section{Publisher's Note}

Springer Nature remains neutral with regard to jurisdictional claims in published maps and institutional affiliations. 